\title{
Transmission through stacked 2D periodic distributions of square conducting patches
}

\author{
Chandra S. R. Kaipa, ${ }^{1, a)}$ Alexander B. Yakovlev, ${ }^{1, b)}$ Francisco Medina, ${ }^{2, c)}$ \\ and Francisco Mesa, ${ }^{3, \mathrm{~d})}$ \\ ${ }^{1}$ Department of Electrical Engineering, The University of Mississippi, University, \\ Mississippi 38677-1848, USA \\ ${ }^{2}$ Microwave Group, Department of Electronics and Electromagnetism, University of Seville, \\ Avda. Reina Mercedes s/ $n$, Seville 41012, Spain \\ ${ }^{3}$ Microwave Group, Department of Applied Physics 1, University of Seville, Avda. Reina Mercedes s/n, \\ Seville 41012, Spain
}

(Received 16 February 2012; accepted 2 July 2012; published online 1 August 2012)

\begin{abstract}
In this paper, we study the transmissivity of electromagnetic waves through stacked twodimensional printed periodic arrays of square conducting patches. An analytical circuit-like model is used for the analysis. The model accounts for the details of the transmission spectrum provided that the period of the unit cell of each patterned layer is well below the wavelength in the dielectric slabs separating the printed surfaces. In particular, we analyze the low-pass band and rejection band behavior of the multilayer structure, and the results are validated by comparison with a computationally intensive finite element commercial electromagnetic solver. The limiting case of an infinite periodic structure is analytically solved and the corresponding band structure is used to explain the passband/stopband behavior of finite structures. In addition, we study in depth the elementary unit cell consisting of a single dielectric slab coated by two metal patch arrays, and its resonance behavior is explained in terms of Fabry-Pérot resonances when the electrical thickness of the slab is large enough. In such case, the concept of equivalent thickness of the equivalent ideal Fabry-Pérot resonator is introduced. For electrically thinner slabs it is also shown that the analytical model is still valid, and its corresponding first transmission peak is explained in terms of a lumpedcircuit LC resonance. (C) 2012 American Institute of Physics. [http://dx.doi.org/10.1063/1.4740054]
\end{abstract}

\section{INTRODUCTION}

The study of electromagnetic waves propagation through periodic structures has a long history, beginning with the introduction of the first diffraction grating ${ }^{1}$ and the first in-depth analysis of such ubiquitous device. ${ }^{2}$ Diffraction gratings for spectroscopy applications are typically devised to operate in the diffraction regime, when the wavelength of the incident radiation is smaller than the grating period. However, more recently, the opposite situation attracted the attention of researchers due to the technological possibility of fabricating structures with extremely fine geometrical details, smaller than the wavelength even at optical frequencies. Thus, the sub-diffraction operation of periodic structures has received considerable attention in various research fields: frequency selective surfaces (FSS) for microwave and millimeter-wave applications (see, for instance, Munk's book $^{3}$ and references therein), design of infrared filters based on periodically patterned metal grids, ${ }^{4,5}$ photonic band-gap structures ${ }^{6,7}$ and, very recently, microwave, $,{ }^{8,9} \mathrm{THz},{ }^{10-13}$ and optical $^{14,15}$ extraordinary transmission systems. Usually, the analysis of this kind of structures relies on the use of commercial full-wave electromagnetic solvers, which generally demand high computation resources for obtaining accurate

\footnotetext{
a)ckaipa@olemiss.edu.

b)yakovlev@olemiss.edu.

c) medina@us.es.

d)mesa@us.es.
}

results. Furthermore, the simulators only provide "numbers" without any physical insight on the nature of the obtained solutions. Fortunately, the modeling of this class of periodic structures operating in the sub-diffraction regime can be carried out, under some circumstances, by means of very simple models based on the elementary distributed circuit theory. For instance, it was already reported ${ }^{16}$ an accurate modeling of extraordinary transmission through electrically thick and highly conductive periodically perforated plates. The same concept has been extended later to other periodic structures, including electrically thick simple or compound gratings, ${ }^{17,18}$ and electrically thin metallic gratings printed on layered dielectric slabs. ${ }^{19-21}$

The transmission spectra of electrically thin gratings or grids are controlled by the dimensions of the grating/grid as well as the thickness and permittivity of the dielectric slabs. However, these parameters provide a limited control of the transmission spectrum, and typically only a narrow transmission band can actually be achieved. A better control of the transmission/reflection spectra is provided by stacking the metal grids or other periodically patterned metal screens. For instance, several metal grids made of narrow perpendicular crossed strips can be arranged parallel to each other and separated by dielectric slabs. This is the principle behind the design of relatively wideband infrared filters ${ }^{4,5}$ and modern wideband microwave FSS. ${ }^{22,23}$ In a recent paper by some of the authors,${ }^{24}$ it has been shown that stacked metal grids separated by dielectric slabs can be accurately analyzed using 
circuit models with the grid parameters known in closed form. That model is restricted to normal incidence and valid for grids having a lattice constant well below the operating wavelength, with a distance between the adjacent grids large enough to avoid higher-order mode interaction. With these limitations, the analytical mode ${ }^{24}$ gives accurate results, and thus it has been used for the explanation of the experimental and numerical data previously reported in Ref. 25. It was discussed in Refs. 24 and 25 that the structure based on stacked metal grids is the microwave analogue to the optical multilayer metal-dielectric stack analyzed in Ref. 26. The metal grids in Ref. 24 behave at microwaves similarly to the thin silver layers with optical negative permittivity in the problem analyzed in Ref. 26. Such structure exhibits a passband behavior that can be explained in terms of coupled modified Fabry-Pérot (FP) resonators using the elementary theory of one-dimensional periodic structures.

In this contribution, we propose an electromagnetic filtering structure that can be considered as a quasicomplementary version of the structure in Refs. 24 and 25. It will be shown that a very accurate analytical model is also available for this system. The proposed structure is formed by a two-dimensional (2D) stack of periodic partially reflective surfaces (PRS) uniformly separated by dielectric slabs. Each PRS consists of a 2D periodic distribution of closely spaced square conducting patches. The separation between two consecutive PRS has to be significantly larger than the transverse gap between conducting patches at each PRS in order to keep negligible the effects of interaction through higher-order modes. Moreover, as in Ref. 24, the period of the $2 \mathrm{D}$ array of patches has to be sufficiently smaller than the wavelength in the involved dielectric media. In a system made up of $N+1$ PRS separated by $N$ identical dielectric slabs, there will appear $N+1$ resonant transmission peaks ranging from zero frequency up to a certain upper limit frequency. It will be shown that this upper frequency limit does not depend on the number of slabs $(N)$. This partially transparent frequency range with total transmission peaks is followed by a deep stopband. After that stopband, a new passband appears. The upper frequency of the low-pass band and the lower and upper frequency limits of the next passband can be analytically obtained from the study of the periodic structure that results from stacking an infinite number of uniformly spaced PRS separated by the dielectric slabs. In contrast to the problem of stacked grids studied in Ref. 24, the electric near field of the structure considered here is very different from that obtained with the circuit model. However, the mean value of the electric field (averaged over the unit cell of the 2D patch array) is accurately accounted for by the analytical model. As shown in the paper, this is sufficient to obtain accurate values for the transmission and reflection coefficients with the circuit model. The above study is directly applicable to the microwave/millimeter-wave/THz regimes if losses are phenomenologically incorporated by adding the appropriate resistors to the model. The model can also give some preliminary insight on the spectrum expected at optical frequencies, when metals are characterized by complex dielectric constants. It should be mentioned that the obtaining of numerical results using full-wave commercial software requires many hours of CPU time (with eventual lacks of convergence) while the analytical model provides results almost instantaneously.

Particular attention is also paid to the study of the simple structure formed by two patch arrays separated by a single dielectric slab. This structure exhibits resonance transmission peaks that can be explained, under certain conditions, in terms of FP resonances of a FP resonator with an equivalent length. This equivalent length can be analytically obtained and is larger than the physical thickness of the dielectric slab provided that the slab is electrically thick enough. For the case of electrically thin dielectric slabs, there appear low frequency resonances that are modeled in terms of quasilumped resonators. In contrast with the electrically thick case, the higher-order resonances of the FP type correspond to a FP resonator having a length smaller than the separation between the PRS.

\section{STACKED 2D ARRAYS OF CONDUCTING PATCHES}

An example of the multilayer configuration studied in this paper is shown in Fig. 1, where it can be seen the front view of each of the stacked PRS (consisting of a 2D periodic array of square conducting patches). Although only $5 \times 5$ unit cells along the $x$ and $y$ directions are shown, the structure is assumed infinite in the lateral directions. Each PRS has sub-wavelength dimensions such that the unit cell size,

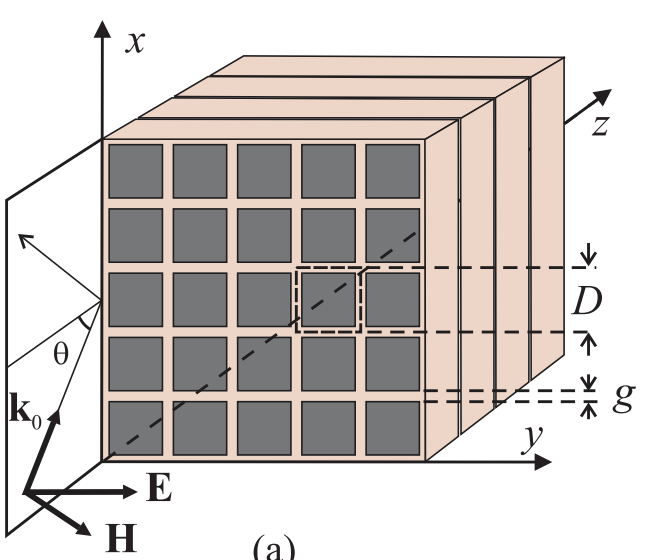

(a)

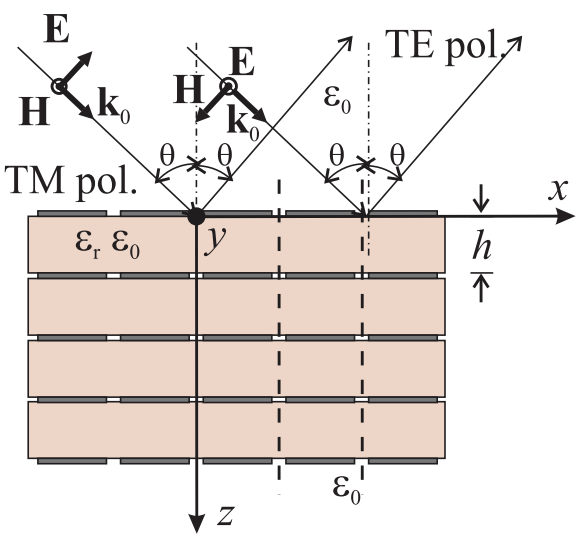

(b)
FIG. 1. Schematics of stacked identical 2D arrays of square conducting patches (dark gray) printed on uniform dielectric slabs of thickness $h$ (light pink). (a) Front view of 25 cells of the structure and (b) cross-section along the direction normal to the PRS. The incidence plane is the $x z$-plane and two orthogonal polarizations (TE and TM) are considered independently. The lattice parameter is $D$ and the gap between the patches is $g$. The thickness of the metal patches is neglected. An elementary unit cell is highlighted with the dashed lines. 
$D$, is smaller than the wavelength in the dielectric slabs at the operation frequency, and the square metal patches occupy most of the surface of the unit cells (i.e., $g \ll D$, where $g$ is the gap between the patches). As shown in Fig. 1(b), $N+1$ PRS of this kind ( $N=4$ in the present example) are stacked and separated by identical dielectric slabs (which can also be air-filled regions). The structure is illuminated with a uniform transverse electromagnetic (TEM) plane wave under oblique incidence conditions ( $\theta$ is the angle formed by the wave vector and the unit vector normal to the surface). Since the structure is isotropic with respect to any direction perpendicular to $z$ (the coordinate along which the structure is stacked), the incidence plane can be arbitrarily chosen. Without loss of generality, the plane of incidence is taken as one of the principal planes of the structure (for example, the $x z$-plane in Fig. 1). Two different polarizations are considered independently: transverse electric (TE) or $s$-polarization and transverse magnetic (TM) or $p$-polarization. The TE case with the electric field perpendicular to the plane of incidence is shown in Fig. 1(a). A single isolated freestanding array of square patches behaves as a PRS with the magnitude of the transmission coefficient monotonically decreasing from unity to very small values in the frequency region of interest (see, for instance, Ref. 4). This behavior is opposite to the one exhibited by the complementary structure: an electrically dense grid made with narrow conducting crossed strips (as it is apparent from Babinet's principle, the reflection and transmission coefficients of the patches and grid structures are interchanged for free-standing structures). In this paper, it is shown that the behavior of a single PRS made of square conducting patches is drastically modified if several PRS of this type are stacked between dielectric slabs (or air-filled regions). This study could have been done using any commercial full-wave electromagnetic solver, but we show that very accurate results can be obtained for the transmission/reflection coefficients using the fully analytical model proposed in this paper. The model is valid for normal and oblique incidence and its accuracy is validated through comparison with computationally intensive full-wave results obtained with the well-known HFss package. ${ }^{27}$

\section{A. Derivation of the analytical circuit model}

Assuming that the wavelength in free space is larger than the period in the transverse direction of the structure under study shown in Fig. $1\left(\lambda_{0}>D\right)$, no diffraction lobes appear and a single plane wave is reflected or transmitted into the far-field region. The phase and amplitude of the transmitted and reflected plane waves depend on the level of excitation of the evanescent fields scattered by each patch array (which account for the near field around the patches). In the absence of patches, these evanescent fields are not excited and the corresponding plane-wave incidence problem in the layered structure can be written in terms of cascaded transmission-line sections characterized by the appropriate propagation constants $(\gamma=\mathrm{j} \beta)$ and characteristic admittances $(Y)$. Following Ref. 28, the values of the propagation constants and characteristic admittances for the air (subscript
0 ) and dielectric (subscript $d$ ) regions, and for TM and TE polarizations are given by

$$
\begin{gathered}
\beta_{0}=k_{0} \sqrt{1-\sin ^{2} \theta} \quad \beta_{d}=k_{0} \sqrt{\varepsilon_{r}-\sin ^{2} \theta}, \\
Y_{0}^{\mathrm{TE}}=\frac{\cos \theta}{\eta_{0}} \quad Y_{0}^{\mathrm{TM}}=\frac{1}{\eta_{0} \cos \theta}, \\
Y_{d}^{\mathrm{TE}}=\frac{\sqrt{\varepsilon_{r}-\sin ^{2} \theta}}{\eta_{0}} \quad Y_{d}^{\mathrm{TM}}=\frac{\varepsilon_{r}}{\eta_{0} \sqrt{\varepsilon_{r}-\sin ^{2} \theta}},
\end{gathered}
$$

where $k_{0}=\omega / c$ ( $\omega$ is the angular frequency and $c$ is the speed of light in vacuum), $\eta_{0}=\sqrt{\mu_{0} / \varepsilon_{0}}$ (free-space impedance), and $\varepsilon_{r}$ is the relative permittivity of the dielectric slabs. When the patches are present, the equivalent transmission-line sections that replace each of the dielectric regions can still be used with a slightly different meaning. Each unit cell of the periodic problem, induced by the presence of the patches, can be viewed as a generalized transmission line whose walls are electric, magnetic, or periodic boundary conditions, depending on the polarization of the impinging wave and the angle of incidence (see, for instance, Ref. 16 for the particular case of normal incidence). Thus, the unit cell highlighted in Fig. 1 (black dashed lines) is represented in Figs. 2(a) and 2(b). For square unit cells, the

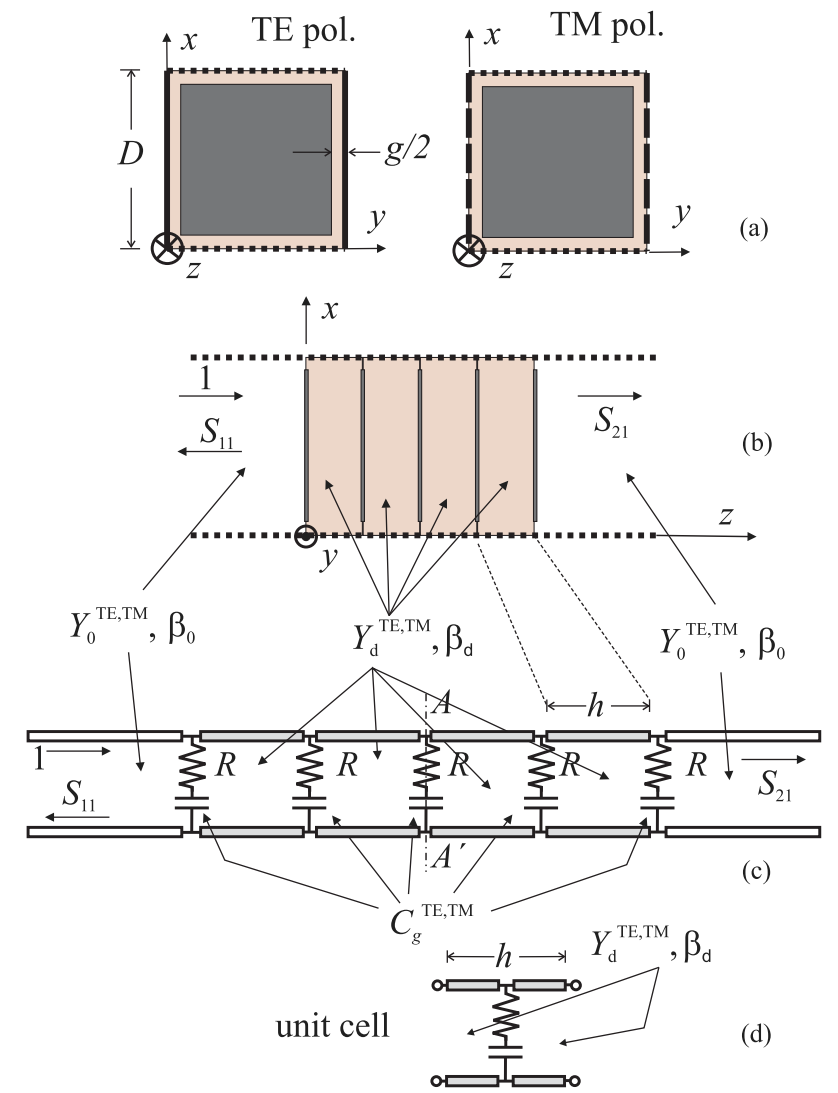

FIG. 2. (a) Front view and (b) side view of the equivalent transmission lines for TE and TM polarized waves. Periodic boundary conditions are applied in the $x$ axis (dotted lines) while electric walls (solid lines; TE polarization) or magnetic walls (dashed lines; TM polarization) are used in the $y$ axis. The equivalent circuit proposed in this paper is depicted in (c). The capacitances of the three internal patches (having dielectric slabs at both sides) are different from the first and the last capacitances (see the main text). (d) Unit cell of the periodic structure along the $z$ direction for an infinite number of slabs $(N \rightarrow \infty)$. 
characteristic admittances of these virtual waveguides are identical to those given in (1b) and (1c) (if the unit cell is rectangular, the aspect ratio can be included in the definition of the characteristic admittances in a trivial manner). As long as the higher-order fields scattered by the patches are evanescent, the presence of the patches can be accounted for by means of properly defined lumped elements, as typically done in microwave modeling of discontinuities in waveguides. This methodology was successfully used in Ref. 24 to deal with stacked grids made with narrow crossed metal strips. Grids are inductive discontinuities but patches are mostly capacitive in nature. Therefore, a suitable circuit model for the structure under study is the one depicted in Fig. 2(c). The global effect of the patches is accounted for by the shunt capacitors and the resistors located between the transmission-line sections (the resistors account for ohmic losses in the skin effect regime). Strictly speaking, inductors should have also been included in the circuit model to take into account higher-order evanescent TE modes. However, since we are assuming electrically small patches occupying most of the unit cell area (i.e., $g \ll D$ ), inductive effects are negligible in the frequency range of interest (see, for instance, Ref. 4). Thus, we only need appropriate expressions for the capacitances and the resistances of the model. An approximate analytical expression for the capacitances can be obtained by using the technique reported in Ref. 29. The following two closed-form expressions are obtained for the two possible polarizations of the impinging wave (TM/TE):

$$
\begin{gathered}
C_{g}^{\mathrm{TM}}=\frac{2 D \varepsilon_{\mathrm{eff}}}{\pi c \eta_{0}} \ln \left[\csc \left(\frac{\pi g}{2 D}\right)\right], \\
C_{g}^{\mathrm{TE}}=\frac{2 D \varepsilon_{\mathrm{eff}}}{\pi c \eta_{0}}\left(1-\frac{\sin ^{2} \theta}{2 \varepsilon_{\mathrm{eff}}}\right) \ln \left[\csc \left(\frac{\pi g}{2 D}\right)\right],
\end{gathered}
$$

where $\varepsilon_{\text {eff }}$ is the average of relative permittivities at both sides of the patch surface $\left(\varepsilon_{\mathrm{eff}}=\left(1+\varepsilon_{r}\right) / 2\right.$ for the first and last PRS, and $\varepsilon_{\mathrm{eff}}=\varepsilon_{r}$ for the internal PRS). Provided that the skin depth, $\delta=\sqrt{2 /\left(\omega \mu_{0} \sigma\right)}$, is meaningfully smaller than the thickness of the metalizations of conductivity $\sigma$, the value of $R$ used in this paper is given by the following simple expression:

$$
R=\frac{D}{(D-g) \sigma \delta} .
$$

According to the proposed model, the obtaining of the reflectivity and transmissivity of the stacked structure in Fig. 1 reduces to the computation of the scattering parameters, $S_{11}$ and $S_{21}$, of the transmission line circuit shown in Fig. 2(c). This is a simple textbook problem of microwave engineering. ${ }^{30}$ In the present case, we can exploit the symmetry of the structures with respect to the $A A^{\prime}$ plane (see Fig. 2(c)). If an even/odd symmetrical excitation is considered, the $A A^{\prime}$ plane becomes a magnetic/electric wall. A reflection coefficient can be obtained for each of those cases ( $S_{11}^{e, o}$ for even/ odd excitations), and the scattering parameters of the original structure can finally be calculated using the superposition principle:

$$
S_{11}=\frac{1}{2}\left(S_{11}^{e}+S_{11}^{o}\right) ; \quad S_{21}=\frac{1}{2}\left(S_{11}^{e}-S_{11}^{o}\right) .
$$

The transmission-line problems defining $S_{11}^{e, o}$ are shown in Fig. 3. These reflection coefficients are different for each polarization (TE or TM), and are related to the input admittances, $Y_{\text {in }}^{e, o}$, shown in Fig. 3 as follows:

$$
S_{11(\mathrm{TE}, \mathrm{TM})}^{e, o}=\frac{Y_{0}^{\mathrm{TE}, \mathrm{TM}}-Y_{\mathrm{in}}^{e, o}}{Y_{0}^{\mathrm{TE}, \mathrm{TM}}+Y_{\mathrm{in}}^{e, o}},
$$

where the input impedances $Y_{\text {in }}^{e, o}$ are easily obtained using well-known admittance translation formulas (see, for instance, Ref. 30). A simple iterative expression for the input admittances can be written for an arbitrary number of slabs. If the number of slabs is odd (instead of even, as in Fig. 3), the last transmission-line section would have a length of $h / 2$ and would be terminated with a short circuit (odd excitation) or an open circuit (even excitation). In any case, the final result is a closed-form expression for the reflection and transmission coefficients of the stacked structure. This approximate expression is validated in Sec. II B by comparison with full-wave numerical results.

\section{B. Validation of the circuit model}

Since a number of approximations have been done to give the circuit model described above, this model will be now validated by proper comparison with full-wave results. The full-wave results in this paper are calculated by using the commercial electromagnetic finite elements solver HFSS. ${ }^{27}$ The comparison starts with a sample structure consisting of five square patch-type PRS made of $18 \mu \mathrm{m}$ thick copper $\left(\sigma_{\mathrm{Cu}}=5.7 \times 10^{7} \mathrm{~S} / \mathrm{m}\right)$ printed on the commercially available Rogers RO3010 substrate (thickness, $h=2.0 \mathrm{~mm}$, $\varepsilon_{r}=10.2$, loss tangent, $\left.\tan \delta=0.0035\right)$. The lattice constant along the $x$ and $y$ directions is $D=2.0 \mathrm{~mm}$ and the gap between patches is $g=0.2 \mathrm{~mm}$. In particular, we present numerical values of the transmissivity $\left(|T|^{2}=\left|S_{21}\right|^{2}\right)$ of this

a) Even excitation circuit

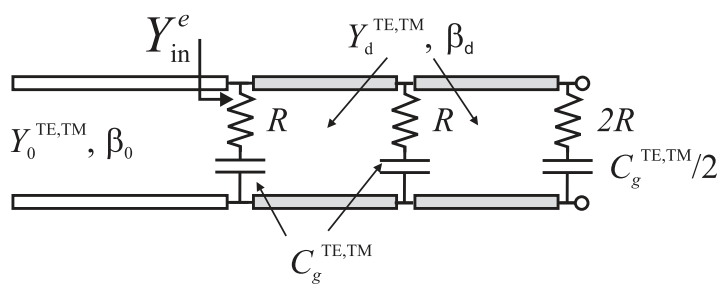

b) Odd excitation circuit

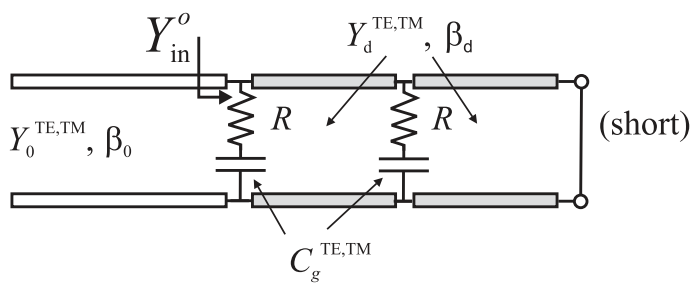

FIG. 3. Equivalent circuits for determining the reflection coefficients under (a) even and (b) odd excitation conditions $\left(S_{11}^{e, o}\right)$ for the structure in Fig. 1. 

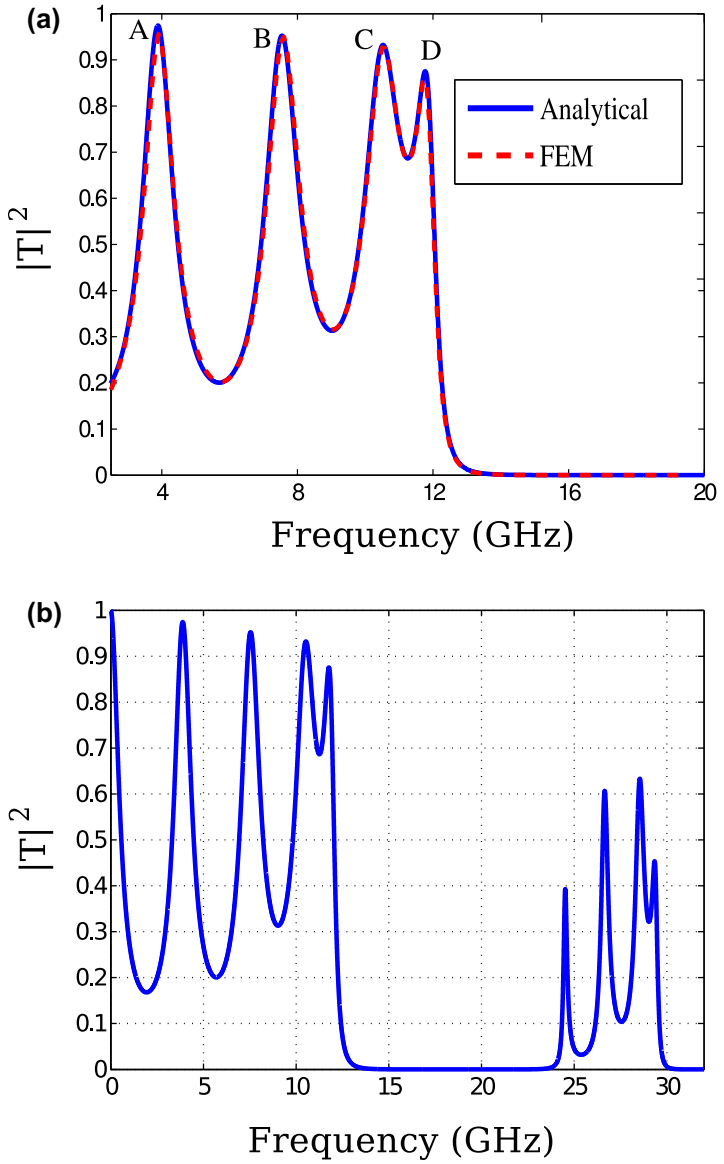

FIG. 4. (a) Comparison between analytical (blue solid lines) and numerical (finite elements method, FEM-HFSS-red dashed lines) results for the transmissivity $\left(|T|^{2}\right)$ of a stacked structure made of 5 PRS separated by 4 dielectric slabs at normal incidence $(\theta=0)$. Dimensions: $D=2.0 \mathrm{~mm}$, $g=0.2 \mathrm{~mm}, h=2.0 \mathrm{~mm}$. Electrical parameters: $\varepsilon_{r}=10.2, \tan \delta=0.0035$, $\sigma_{\mathrm{Cu}}=5.7 \times 10^{7} \mathrm{~S} / \mathrm{m}$. (b) Analytical predictions over a wider frequency band showing a second passband at around $24-30 \mathrm{GHz}$ (numerical data are not included due to convergence problems with HFSs for the high frequency portion of the spectrum).

structure computed with HFSS along with the analytical data provided by our model (dielectric losses have been incorporated in the model by using complex values for the relative dielectric constant in (1a)-(1c)). This quantity is plotted in Fig. 4(a) as a function of frequency at normal incidence (in the present situation of square patches and normal incidence, there is no distinction between TM and TE polarizations).

In the frequency range where we have obtained results from HFSS within an acceptable lapse of time, there is an excellent agreement between numerical and analytical data. In the explored range of frequencies, the structure exhibits a low-pass filter behavior with strong ripples. Apart from the transmission peak occurring at zero frequency (not shown in Fig. 4(a) and also occurring when a single PRS is used), the structure exhibits other four high-transmission peaks (labeled $\mathrm{A}, \mathrm{B}, \mathrm{C}$, and D). Note that, at the maximum frequency considered in that plot $(20 \mathrm{GHz})$, the ratio $D / \lambda_{0} \approx 0.13$ and $D / \lambda_{d} \approx 0.43\left(\lambda_{d}=2 \pi / \beta_{d}\right)$. It is interesting to emphasize that the condition $D \ll \lambda_{d}$ has not to be enforced strictly: $D$ is simply required to be sufficiently smaller than $\lambda_{d}$. Indeed, the model is expected to work if (1) the inductance of the patch is negligible (as mentioned before, this is essentially related to the gap size: $g \ll D$ ) and (2) the frequency dependence of $C_{g}$ can be neglected (the frequency-dependent behavior of $C_{g}$ is here relevant only at frequencies above $20 \mathrm{GHz}$; corresponding to $D \approx 0.75 \lambda_{d}$ ). Similar comparisons have also been done for a wide variety of geometrical and electrical parameters and the same good agreement has been observed. If the analytical model is used up to higher frequencies, Fig. 4(b) shows the appearance of a second passband with four transmission peaks that are more attenuated than in the first transmission band. HFSs data have not been included here because, in that frequency region, we found convergence problems to obtain numerical results with the simulator.

If the transmission spectrum in Fig. 4(b) is compared with the spectra reported in Ref. 25 for the stacked grids, we can see that, in contrast with the bandpass behavior of the grid structure, the patch structure exhibits a low-pass band followed by a wide stopband (followed by another passband, and so on). This behavior is observed independently of the number of stacked layers. This fact is illustrated in Fig. 5(a), where three plots for the transmissivity corresponding to several numbers of layers $(N=2,4$, and 8$)$ are depicted.
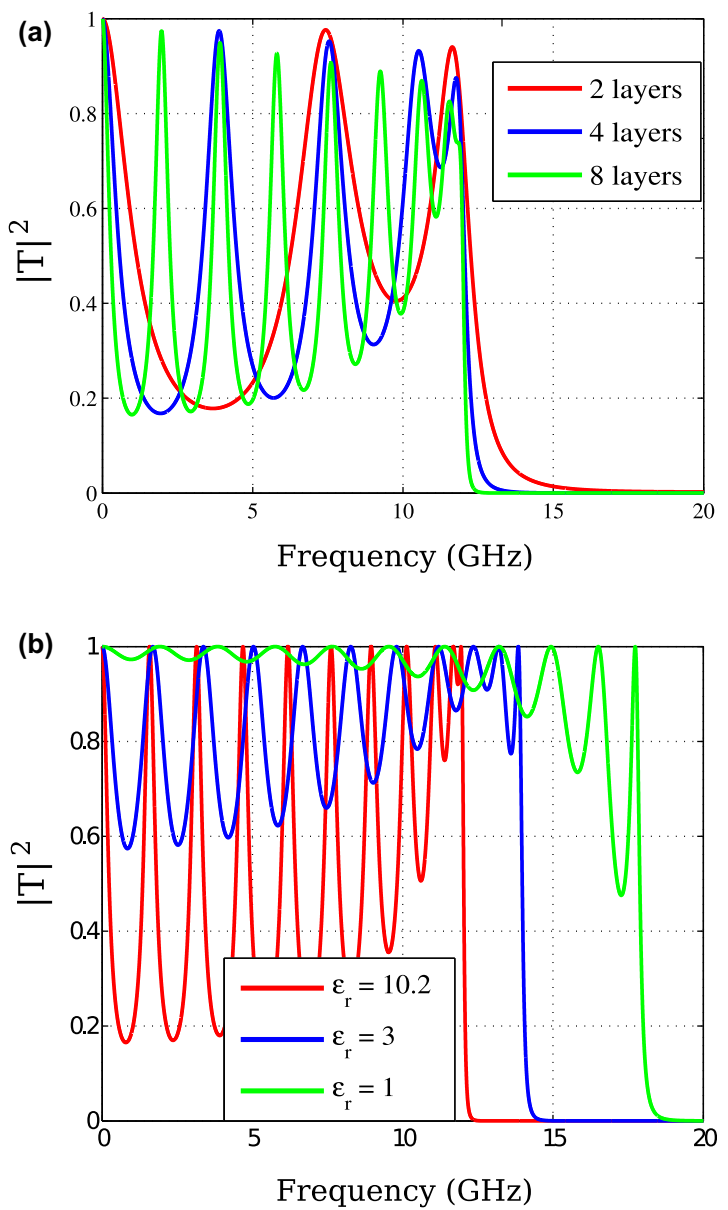

FIG. 5. (a) Transmission spectra obtained for $N=2,4$, and 8 dielectric slabs. Dimensions and electrical parameters are the same as in Fig. 4. (b) Transmission spectra $(N=10)$ for three different values of the dielectric constants of the regions separating the PRS (losses have been ignored). The transverse unit cell dimensions are the same as in Fig. 4 and $h=2.0 \mathrm{~mm}, 4.0 \mathrm{~mm}$, and $6.0 \mathrm{~mm}$ for $\varepsilon_{r}=10.2,3.0$, and 1.0 , respectively. 
Another curve (red) for $N=10$ and the same electrical and dimensional parameters appears in Fig. 5(b) (no losses are included in this case). The number of transmission maxima (excluding the one at zero frequency) is equal to the number of dielectric slabs. However, the upper frequency limit of the low-pass band does not seem to depend meaningfully on the number of layers. The calculated upper frequency limit (at $3 \mathrm{~dB}$ below the maximum) is shown in Table I for an increasing number of slabs.

From this table and Figs. 4 and 5, it is clear that increasing the number of layers results in an increasing number of peaks within a certain fixed frequency band. The upper limit of this band should rather be linked to the geometry and electrical parameters of the unit cell corresponding to a periodic structure along the $z$ direction in the limit $N \rightarrow \infty$. Thus, the frequency regions where transmission is possible can be determined by studying the band structure of the $1 \mathrm{D}$ photonic crystal resulting from cascading along the $z$ direction an infinite number of identical cells such as that represented in Fig. 2(d). It turns out that the eigenvalues for that periodic structure can easily be obtained by using the proposed circuit model. The resulting periodically loaded transmission line supports Bloch waves whose propagation constant can be computed via the method reported in Ref. 30 (this method was also used in Ref. 25 to explain the existence of bands in a stacked-grids structure). Following the procedure described in Ref. 30, the following dispersion equation for the Bloch waves is obtained:

$$
\cos \left(k_{B} h\right)=\cos \phi-\frac{b}{2} \sin \phi,
$$

where $k_{B}$ is the Bloch wavenumber, $\phi=\beta_{d} h$ is the electrical thickness of the slabs, and $b$ is the normalized admittance associated with the capacitances in (2a) or (2b); i.e., $b=\left(\omega C_{g}^{\mathrm{TE}, \mathrm{TM}}\right) / Y_{d}^{\mathrm{TE}, \mathrm{TM}}$. The expression (6) yields a band structure with passbands separated by forbidden frequency regions. The Bloch wavenumber is real (passband) within those frequency regions for which $|\cos \phi-b / 2 \sin \phi|<1$ and purely imaginary in the complementary regions (forbidden bands). The Bloch dispersion curves (first two propagation bands) for the unit cell associated with the structures analyzed in Figs. 4 and 5 and Table I are plotted in Fig. 6.

It can be observed how the low-pass band coincides with the region where the finite structures have nonnegligible transmission (and $N$ high-transmission peaks). The forbidden band in Fig. 6 perfectly accounts for the low transmission region of the finite structures. The Brillouin dia-

TABLE I. Upper frequency limit of the low-pass band of the structure with the dimensions and electrical parameters in Fig. 4 as a function of the number of slabs, $N$.

\begin{tabular}{lc}
\hline \hline$N$ & Upper frequency $(\mathrm{GHz})$ \\
\hline 4 & 11.77 \\
5 & 11.80 \\
6 & 11.82 \\
10 & 11.90 \\
18 & 11.99 \\
30 & 12.02 \\
\hline \hline
\end{tabular}

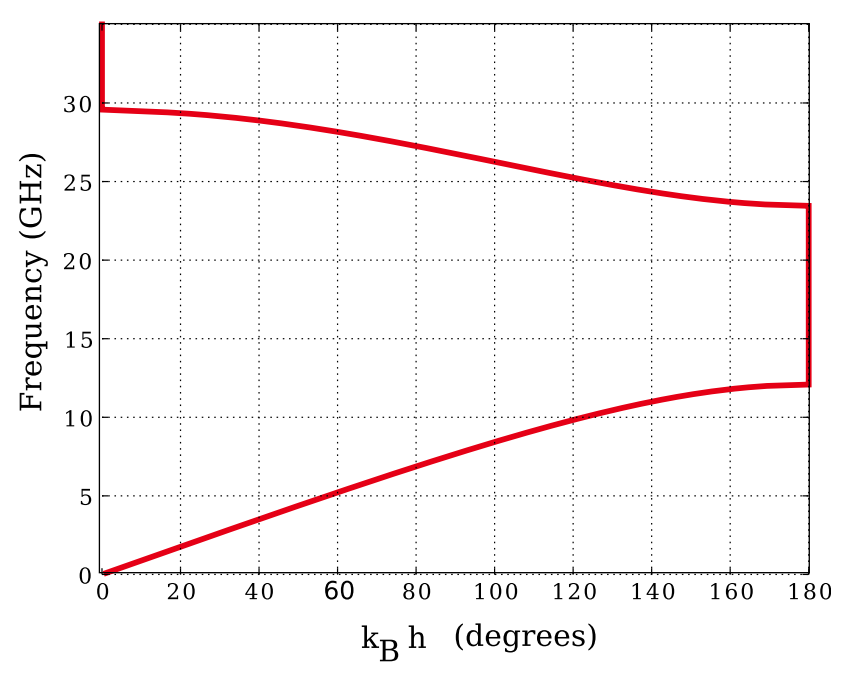

FIG. 6. Brillouin diagram for the first two transmission bands of an infinite periodic structure (1D photonic crystal) with the same unit cell as that used in the curves plotted in Fig. 4. The non-zero transmission region in Fig. 4 matches the first passband in this graph, and the low transmission region in Fig. 4 coincides with the stopband region in this figure. The second passband, which is backward, is consistent with the second set of peaks appearing in Fig. 4(b).

gram suggests the existence of a second transmission band that is clearly anticipated by our circuit model in Fig. 4(b). Note that the first band is forward while the second one is backward.

At this point, it is worth mentioning that, in the same manner that the dense metal grid structures studied in Ref. 25 mimic the behavior of stacked dielectric slabs separated by negative-permittivity solid thin films (metals at optical frequencies have a negative real part of the permittivity), the response of the structure studied in this paper resembles the response of a system of dielectric slabs separated by thin dielectric foils of very high permittivity. In the structures with thin dielectric or metal films separating dielectric slabs, no evanescent TE/TM fields are excited. In such cases, the required stored reactive energy is exclusively associated with the presence of TEM standing waves. On the contrary, the reactive energy in the structures analyzed in this paper and in Ref. 25 comes from TEM standing waves as well as higher-order TE and TM evanescent modes.

It is also interesting to study the behavior of the transmissivity for high and low dielectric constant slabs. In Fig. 5(b), we have plotted the low-pass transmission spectra obtained for three different slabs. The spectra are qualitatively similar but some differences can be appreciated. First, the upper limit of the low-pass band is different for the three cases. One might expect that this upper limit depended only on the electrical thicknesses of the slabs. However, this is not the case: The electrical thickness of the slabs at the upper limit frequency is approximately $0.51 \pi, 0.6 \pi$, and $0.71 \pi$ radians for the high, medium, and low dielectric constant cases, respectively. Actually, Eq. (6) has to be considered to establish such limit. However, another more interesting differential feature is that the ripples level is much higher for high dielectric constant materials than for low permittivity materials. In the limit case of $\varepsilon_{r}=1.0$, ripples are low except for frequencies very close to the upper limit of the low-pass band. This behavior is 
the opposite to the one observed for higher permittivity slabs, for which the ripples depth decreases as the frequency increases up to reach the upper limit of the band. Note that low permittivity slabs would yield better low-pass filters since their frequency response is flatter.

The model proposed in this paper is also valid for oblique incidence. Since the HFSS calculations for several dielectric slabs take a long time, the accuracy of the model for angles of incidence different from zero has been tested for a single slab structure. The comparison between HFss and analytical results for several angles of incidence and TE/TM incidence is shown in Fig. 7. From the plots, it is clear that the model also works quite well for oblique incidence. For TM polarization, the transmission peak is found to be hardly sensitive to the angle of incidence, which is a desirable feature for many applications. However, a slight shift to higher frequencies is observed for increasing values of the incidence angle under TE polarization.

\section{FIELD DISTRIBUTIONS AT THE RESONANCE FREQUENCIES}

In Ref. 24, it was claimed that the field profile along $z$ predicted by the circuit model should agree with that pro-
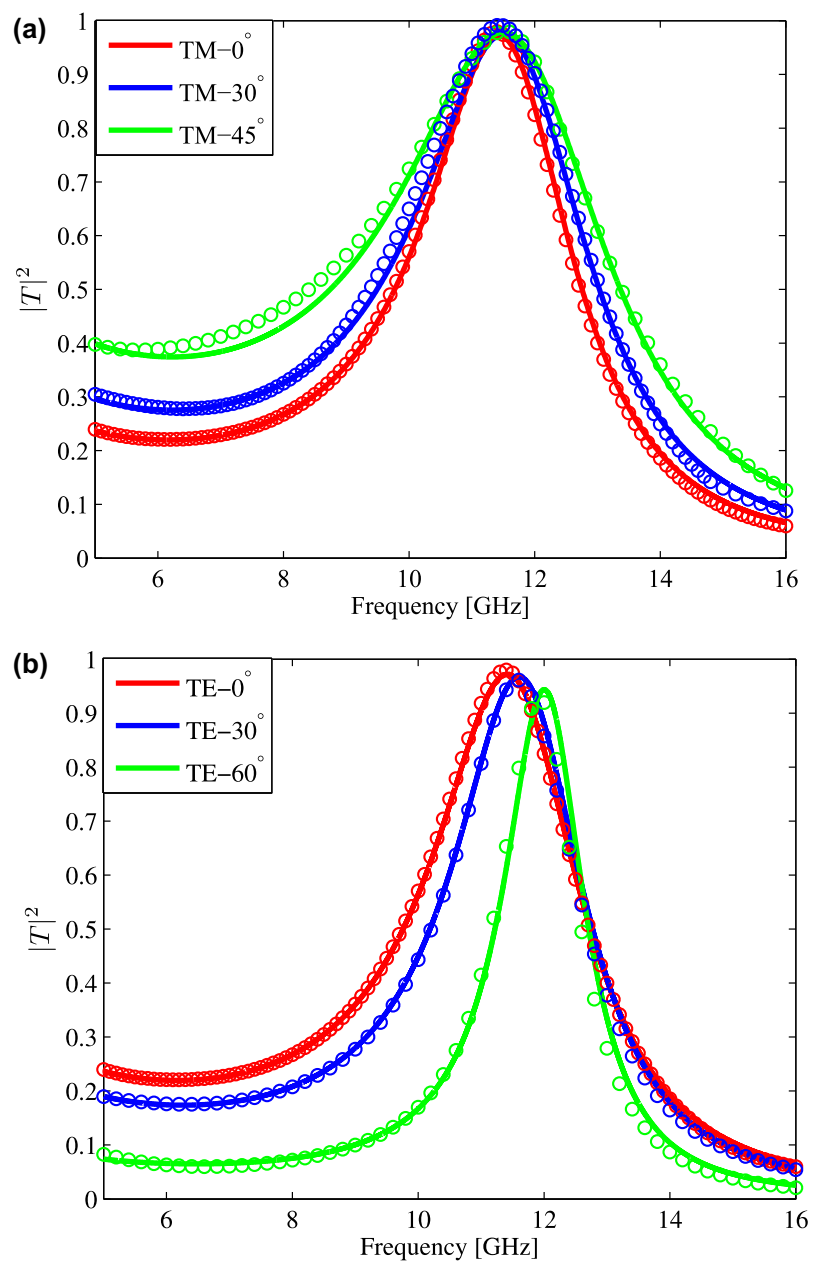

FIG. 7. Transmission curves for a single slab structure $(N=1)$ under oblique TM (a) and TE (b) incidence for several values of $\theta$. Solid lines are analytical results and circles have been obtained with HFSs. The dimensions and the electrical parameters are the same as in Fig. 4. vided by full-wave numerical simulations. Thus, in that paper, it was verified that the voltage distribution along the transmission lines of the proposed circuit model was almost identical to the electric field distribution (computed using HFSS (Ref. 27)) along the central line crossing the structure unit cell. Since the fields predicted by the analytical model and the numerically computed fields were very close to each other, the scattering parameters predicted by the two approaches were clearly expected to be also very similar. However, for the structure considered in the present work, it is clear that we cannot expect the same degree of similarity between the voltage distribution provided by the circuit model and the transverse electric field distribution given by the full-wave numerical simulations. Thus, for instance, the voltage distribution along the transmission-line equivalent system in Fig. 2(c) cannot reproduce the fact of having zero transverse electric field in the perfectly conducting patches (note that the location of the patches corresponds to the location of the capacitors in the circuit model). This fact is illustrated in Fig. 8 for the four frequency points corresponding to the peaks A, B, C, and D in Fig. 4.

In each of the plots in Fig. 8, we have included the $y$-component of the electric field computed with HFSS (Ref. 27) (solid green lines). In all the cases, this field pattern has zeros at those points where the perfectly conducting patches are located. The "average" electric field deduced from the voltage distribution predicted by the circuit model is represented in Fig. 8 as solid blue lines. It is clear that the HFss and the circuit-model field patterns are completely different. However, we already found in Fig. 4(a) that the scattering parameters predicted by the analytical model are very accurate. To solve this apparent paradox, we should consider that the scattering parameters are power-related quantities and, therefore, they should be accurately computed from a good estimation of the average electric field over the cross-section of the unit cell. The longitudinal $(z)$ profile of this average field, which could be called macroscopic field, is very close to the actual longitudinal profile of the local field (or microscopic field) in the case of the grid structures studied in Ref. 24. However, the longitudinal profiles of the macroscopic and microscopic fields are completely different in the patches structure considered here. The average full-wave field pattern can easily be computed from HFSS data, and its value has been included in Fig. 8 as dashed red lines. Since this average field is controlled by the TEM component of the total field (the average value of any higher-order mode is zero), its longitudinal profile is found to be almost identical to the voltage profile given by the circuit model. This good agreement is what finally explains the accuracy of the scattering coefficients provided by the circuit model.

The longitudinal profile of the average field pattern for each of the resonances depicted in Fig. 4 has distinctive features that allow us to associate each pattern with each resonance. The lowest resonance frequency pattern (Fig. 8(a)) has a single zero over the central PRS. The second one (Fig. 8(b)) has two zeros in the positions corresponding to the second and fourth patch arrays. The third resonance frequency pattern (Fig. 8(c)) has three zeros: one over a patch array and the other two in the middle of the first and fourth slabs. Finally, 

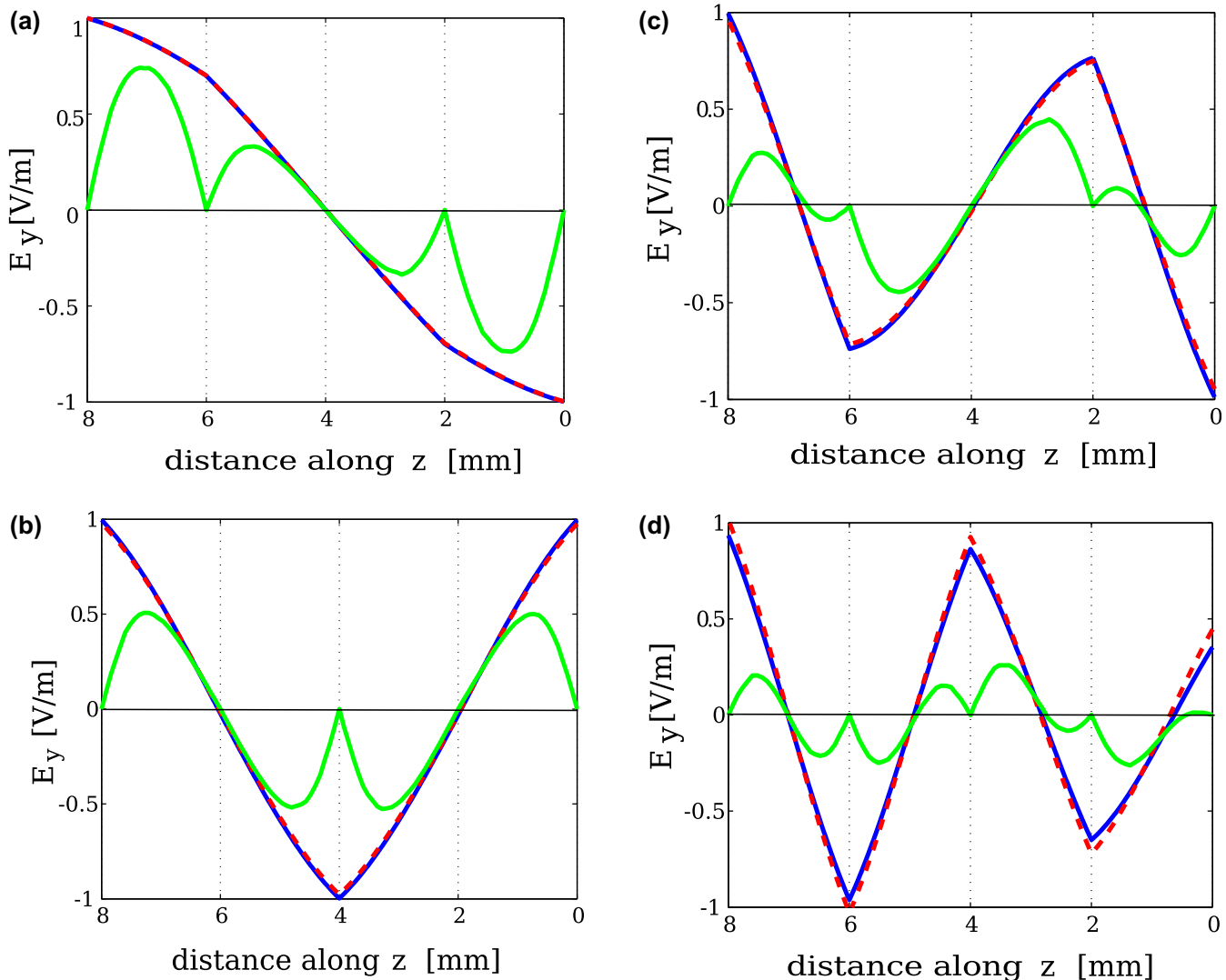

FIG. 8. Longitudinal profile of the $y$-component of the electric field for the frequencies corresponding to the transmission peaks plotted in Fig. 4 ((a) A; (b) B; (c) C; (d) D). Solid green lines, the detailed local field computed by HFss along a center line across the structure; dashed red lines, the corresponding average electric field along every transverse cross-section; solid blue lines, the electric field extracted from the analytical circuit model.

the highest resonance frequency field pattern (Fig. 8(d)) has four zeros: each one inside of one of the four dielectric slabs. This rule for the field patterns applies to any number of slabs.

\section{THE BASIC STRUCTURE: TWO PRS SEPARATED BY A DIELECTRIC SLAB}

In this final section, we study in depth the simplest version of the structure considered in this paper with the purpose of obtaining a deeper understanding of the type of resonances that are possible in this structure. The simplest stacked structure corresponds to the case $N=1$. Two identical 2D arrays of perfectly conducting square patches are printed and aligned on both sides of a single dielectric slab. In the absence of patches, the slab behaves as a Fabry-Pérot resonator exhibiting transmission peaks at those frequencies for which the thickness of the slab is an integer number of half-wavelengths (or, equivalently, $\beta_{d} h=n \pi, n= \pm 1, \pm 2, \ldots$ ). The presence of the patches modifies the situation. Following the theory in Sec. II, the equivalent circuit for the single slab with patches at both sides would be a section of transmission line of length $h$ (wavenumber $\beta_{d}$ and characteristic admittance $Y_{d}^{\mathrm{TE}, \mathrm{TM}}$ ) inserted between two transmission lines having wavenumber $\beta_{0}$ and characteristic admittance $Y_{0}^{\mathrm{TE}, \mathrm{TM}}$ (see (1a)-(1c)). The resulting equivalent circuit is formally identical to the one used in Ref. 17 to analyze electrically thick slit gratings. In that paper, the resonances were explained in terms of modified FP resonators with an equivalent length slightly larger than the physical length of the resonator (in that case, the depth of the slit). However, although the equivalent circuit is identical to the one in Ref. 17, the values of the parameters now involved in the circuit model could be drastically different from those in Ref. 17. This fact can give rise to a completely different operation. An implicit equation for the location of the resonance frequencies (transmission peaks) was derived in Ref. 17 from the circuit model. Once adapted to the notation in this paper, that equation can be written as

$$
\tan \left(\beta_{d} h\right)=\mathrm{j} \frac{2 Y_{d} Y_{C}}{Y_{d}^{2}-Y_{0}^{2}+Y_{C}^{2}}=-\frac{2 \omega Y_{d} C_{g}}{Y_{d}^{2}-Y_{0}^{2}-\left(\omega C_{g}\right)^{2}},
$$

where the upper indexes for TE and TM have been suppressed for simplicity. Although the previous equation should be numerically solved, some qualitative ideas can help us to understand the different type of solutions that are expected. The left hand side in (7) is a tangent function having poles at those frequency values making $\beta_{d} h=(2 n+1) \pi / 2$ and zeros at those frequency values where $\beta_{d} h=n \pi(n=0,1,2, \ldots)$ is satisfied. At the right hand side in (7), we have a rational function whose single positive pole is located at $\omega_{p}=\left(Y_{d}^{2}-Y_{o}^{2}\right)^{1 / 2} / C_{g}$. For the typical values of the parameters of (7) considered in Ref. 17, the position of this pole was well beyond the frequency range of interest, and thus the rational function behaved as a linear function of $\omega$ with a small negative slope within the frequency range of interest. The crossing between this almost-linear function and the tangent function is expected to occur below and close to the zeros of the tangent function. A similar situation is found for 
the present structure when the pole of the rational function is above one or several of the roots of the tangent function (i.e., when $\omega_{p}>n \pi c /\left[h \sqrt{\varepsilon_{r}-\sin ^{2} \theta}\right]$ for some $\left.n>1\right)$. In such a case, following the discussion in Ref. 17, the transmission peaks occurring below $\omega_{p}$ can be approximately explained in terms of FP resonances of an equivalent slab having a thickness larger than the physical one. This equivalent thickness, $h_{\text {eq }}$, is given by the following expression:

$$
h_{\mathrm{eq}}=h+\Delta h=h+\frac{2 c C_{g}}{Y_{d}} .
$$

The above expression is useful for relatively electrically thick slabs since, in such cases, the tangent function has several poles and zeros below the pole of the rational function in (7). The field patterns inside the dielectric slabs then correspond to standing waves having one or more maxima and zeros, such as expected for FP-like resonances. However, due to the relatively high values of $C_{g}$ in the present problem (when compared with the corresponding values in Ref. 17) and the possibility of having low values of $\left(Y_{d}^{2}-Y_{0}^{2}\right)$ (low permittivity slabs), the pole of the rational function can be below the first positive root of the tangent function or even below the first pole of such function (i.e., $\omega_{p}<c \pi /\left[h \sqrt{\varepsilon_{r}-\sin ^{2} \theta}\right]$ ). A simple inspection of the graphical representation of the tangent and rational functions tells us that, in this latter case, there is a solution to (7) for a frequency value located between $\omega_{p}$ and the frequency corresponding to $\beta_{d} h=\pi / 2$. At such resonance frequency, the electrical thickness of the slab is less than $\lambda_{d} / 4$ and no FP mechanism can be invoked. This is the situation found for electrically short slabs, where the unit cell behaves more like a quasi-lumped resonator. The electric field is strongly concentrated around the gaps oriented perpendicularly to the impinging electric field, while the cavity between the two PRS mainly stores magnetic energy.

In order to illustrate the application of the concept of equivalent thickness and its range of validity, we have calculated the position of the first resonance frequency for a single slab of relative dielectric constant $\varepsilon_{r}=10.2$ coated by square metal patches (having dimensional parameters $D=2.0 \mathrm{~mm}$ and $g=0.2 \mathrm{~mm}$ for several values of the slab thickness, $h$ ). The results have been included in Table II, where the numerical HFSS data are compared versus data obtained using the circuit formula (7) and the approximate equivalent thickness formula (8). It is clear from the table that the circuit model

TABLE II. Comparison of the frequencies of total transmission, $f_{\mathrm{TT}}$, calculated by solving the dispersion equation (7), the equivalent thickness formula (8), and using the full-wave HFss solver. The analyzed structure is a two-sided patch array $(D=2.0 \mathrm{~mm} ; g=0.2 \mathrm{~mm})$ printed on a dielectric slab $\left(\varepsilon_{r}=10.2\right)$ for different thicknesses under normal incidence conditions.

\begin{tabular}{lccc}
\hline \hline$h(\mathrm{~mm})$ & $f_{\mathrm{TT}}(\mathrm{GHz})$ via $(7)$ & $f_{\mathrm{TT}}(\mathrm{GHz})$ via $(8)$ & $f_{\mathrm{TT}}(\mathrm{GHz})$ via HFSS \\
\hline 1 & 17.211 & 13.0606 & 16.68 \\
2 & 11.425 & 10.2174 & 11.4 \\
4 & 7.279 & 7.1182 & 7.23 \\
6 & 5.458 & 5.4616 & 5.40 \\
8 & 4.392 & 4.4304 & 4.38 \\
10 & 3.686 & 3.7268 & 3.65 \\
\hline \hline
\end{tabular}

provides accurate results for any thickness of the slabs, although the prediction for the case $h=1.0 \mathrm{~mm}$ has poorer accuracy (in this case the interaction between consecutive PRS through higher-order modes is not negligible). However, the prediction given by (8) clearly fails for electrically thin slabs.

In Fig. 9(a), the HFSS and circuit model predictions for relatively thin slabs $(h \leq 2.0 \mathrm{~mm})$ are shown for comparison purposes. This figure makes apparent that the circuit model becomes less accurate as the value of $h$ decreases. However, the quality of the circuit model description for a given value of $h$ is better for small values of $g$, as illustrated in Fig. 9(b). This observation is consistent with the fact that higher-order modes are less important when accounting for the interactions between PRS when the gaps are small.

Coming back to the analysis of the results in Table II, we can deduce that, even though the circuit model description works quite well, the equivalent-thickness slab concept starts to lose its meaning for $h \$ 4.0 \mathrm{~mm}$. Indeed, the first resonance (total transmission) frequencies for $h=1.0 \mathrm{~mm}$ and $h=2.0 \mathrm{~mm}$ correspond to the values of electrical thickness of the dielectric slabs below $\pi / 4$ and, hence, these first two cases cannot be associated with FP resonances but rather with
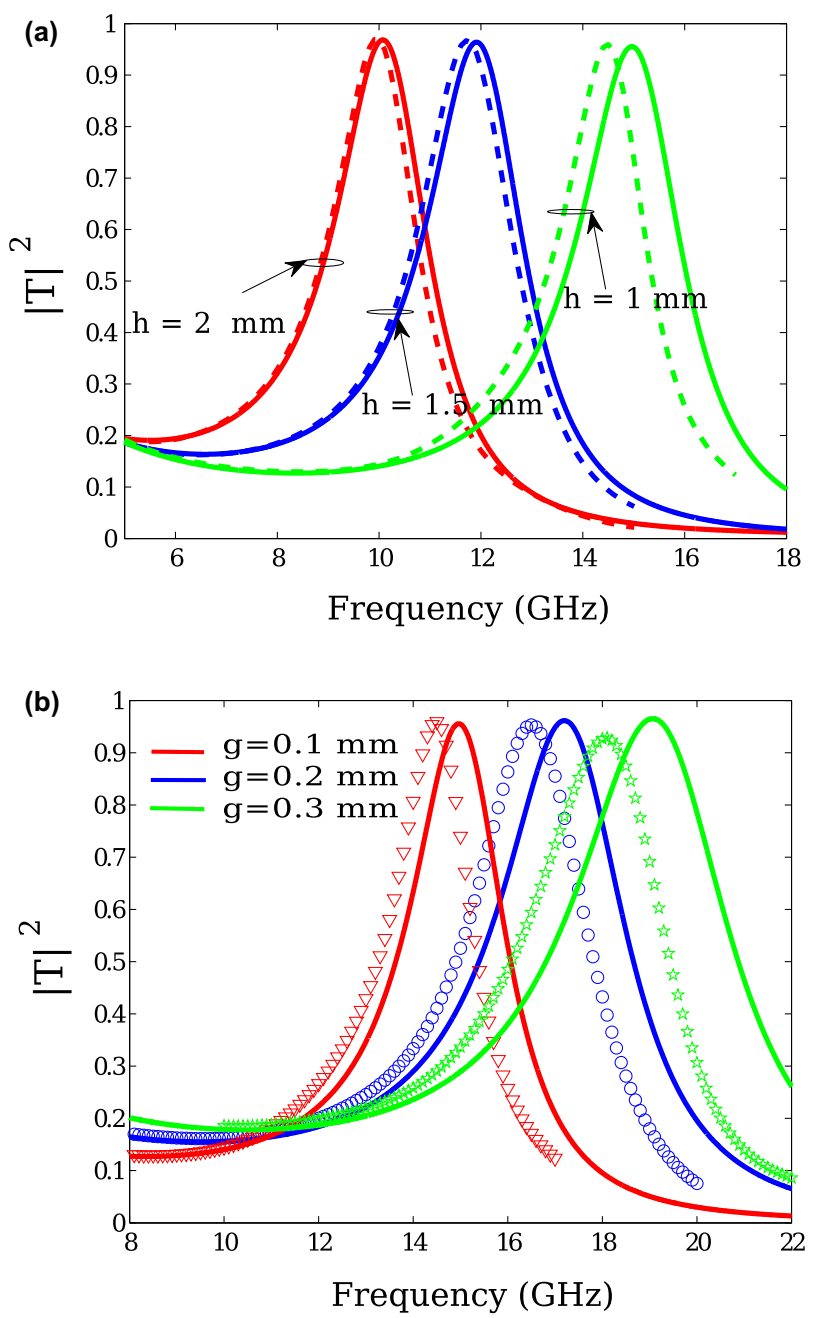

FIG. 9. (a) Comparison between circuit model and HFSs predictions around the first resonance frequency for three different slab thicknesses $\left(\varepsilon_{r}=10.2\right.$, $h=1.0,1.5$, and $2.0 \mathrm{~mm}$ ). (b) The same comparison (case $h=1.0 \mathrm{~mm}$ ) for three different gaps between the patches $(g=0.1,0.2$, and $0.3 \mathrm{~mm})$. 

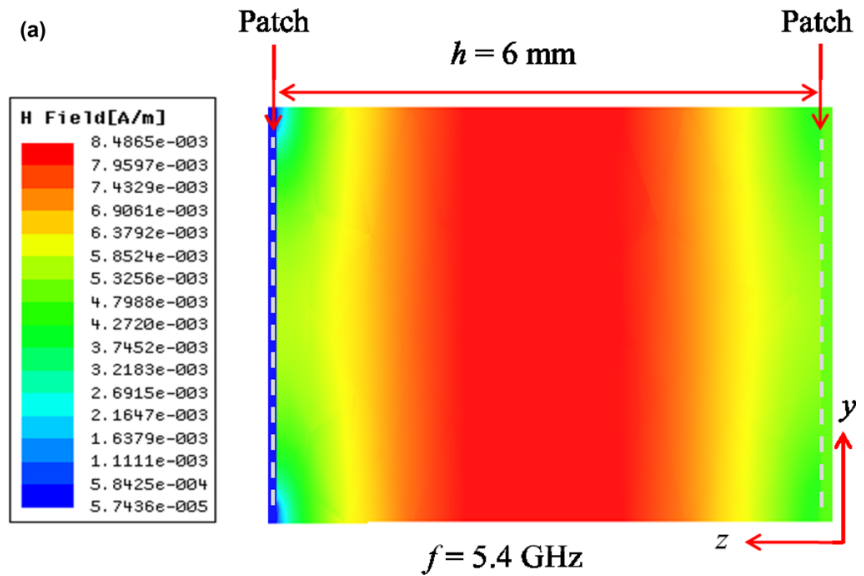

(b)
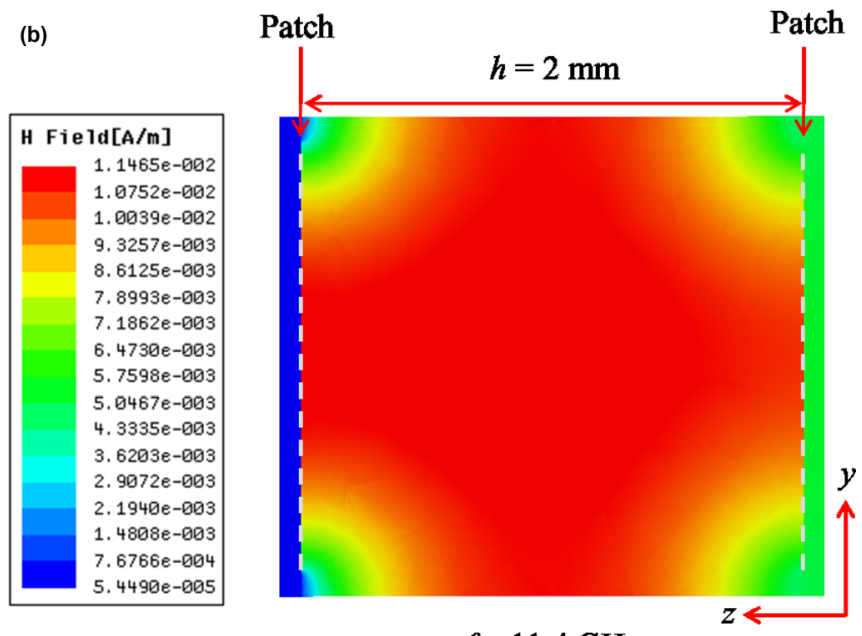

$f=11.4 \mathrm{GHz}$

FIG. 10. (a) Magnetic field color map for the first resonance frequency in the case $h=6.0 \mathrm{~mm}$ (see Table II). (b) The same plot for $h=2.0 \mathrm{~mm}$. (see Table II and Fig. 9).

quasi-lumped resonances. In order to understand the difference between the two situations (FP-like resonances versus quasi-lumped resonances), we examine the distribution of the magnetic field at resonance. Thus, Figs. 10(a) and 10(b) show the magnetic field distribution inside the dielectric region for the first resonance frequency obtained for $h=6.0 \mathrm{~mm}$ and $h=2.0 \mathrm{~mm}$, respectively. In the first case, a typical sinusoidal pattern of FP type is visualized. However, in the second case $(h=2.0 \mathrm{~mm})$, the magnetic field distribution is much more uniform. A similar plot for the electric field shows a similar pattern, with a very strong electric field around the gaps, as expected. For the electrically thin slab case shown in Fig. 10(b), the resonance frequency can be estimated from the local capacitances (lumped capacitances of the gaps) and the overall inductance of the unit cell section between the PRS, thus validating our consideration of this resonance as a quasi-lumped one.

\section{CONCLUSION}

In this work, it has been shown that the study of the wave propagation along stacked partially reflecting surfaces consisting on square closely spaced metal patches separated by dielectric slabs can be carried out analytically with negligible computational effort by means of a very simple circuit model. The parameters of the model are known in closed form. Using this model, the band configuration of the periodic structure resulting of stacking an infinite number of PRS and slabs can be obtained. A first forward low-pass band is followed by a stopband region after which a backward-wave passband appears. This band configuration provides important information about the distribution of the transmission peaks of a realistic structure having a finite number of PRS and slabs. The simplest case with only one dielectric slab has been studied in depth and two types of resonances have been identified: quasi-lumped and Fabry-Pérot like resonances. The methodology used in this paper can be useful in the design of devices based on these structures, for which numerical simulations are cumbersome or even nonconvergent.

\section{ACKNOWLEDGMENTS}

F. Medina and F. Mesa acknowledge financial support from Spanish Ministry of Science and Innovation, European Union FEDER funds, and Spanish Junta de Andalucía (Projects TEC2010-16948, Consolider EMET CSD2008-00066, and P09-TIC-4595).

${ }^{1}$ D. Rittenhouse, Trans. Am. Philos. Soc. 2 , 37 (1786).

${ }^{2}$ J. Fraunhofer, Ann. D. Phys. 74, 337 (1823).

${ }^{3}$ B. A. Munk, Frequency Selective Surfaces: Theory and Design (Wiley, 2000).

${ }^{4}$ R. Ulrich, Infrared Phys. Technol. 7, 37 (1967).

${ }^{5}$ R. Ulrich, Infrared Phys. Technol. 7, 65 (1967).

${ }^{6}$ E. Yablonovitch, Phys. Rev. Lett. 58, 2059 (1987).

${ }^{7}$ S. John, Phys. Rev. Lett. 58, 2486 (1987).

${ }^{8}$ M. Beruete, M. Sorolla, M. Navarro-Cía, F. Falcone, I. Campillo, and V. Lomakin, Opt. Express 15, 1107 (2007)

${ }^{9}$ M. Navarro-Cía, M. Beruete, F. Falcone, J. M. Illescas, I. Campillo, and M. Sorolla, IEEE Trans. Antennas and Propagat. 59, 2980 (2011).

${ }^{10}$ S. A. Kuznetsov, M. Navarro-Cía, V. V. Kubarev, A. V. Gelfand, M. Beruete, I. Campillo, and M. Sorolla, Opt. Express 17, 11730 (2009).

${ }^{11}$ J. Carbonell, C. Croenne, F. Garet, E. Lheurette, J. L. Coutaz, and D. Lippens, J. Appl. Phys. 108, 014907 (2010).

${ }^{12}$ J. Wu, H. Dai, H. Wang, B. Jin, T. Jia, C. Zhang, C. Cao, J. Chen, L. Kang, W. Xu, and P. Wu, Opt. Express 19, 1101 (2011).

${ }^{13}$ M. Choi, S. H. Lee, Y. Kim, S. B. Kang, J. Shin, M. H. Kwak, K.-Y. Kang, Y.-H. Lee, N. Park, and B. Min, Nature 470, 369 (2011).

${ }^{14}$ T. W. Ebbesen, H. J. Lezec, H. F. Ghaemi, T. Thio, and P. A. Wolff, Nature (London) 391, 667 (1998)

${ }^{15}$ C. García-Meca, J. Hurtado, J. Martí, and A. Martínez, Phys. Rev. Lett. 106, 067402 (2011)

${ }^{16}$ F. Medina, F. Mesa, and R. Marqués, IEEE Trans. Microwave Theory Tech. 56, 3108 (2008).

${ }^{17}$ F. Medina, F. Mesa, and D. C. Skigin, IEEE Trans. Microwave Theory Tech. 58, 105 (2010).

${ }^{18}$ R. Yang, R. Rodríguez-Berral, F. Medina, and Y. Hao, J. Appl. Phys. 109, 103107 (2011).

${ }^{19}$ R. Rodríguez-Berral, F. Medina, and F. Mesa, Appl. Phys. Lett. 96, 161104 (2010).

${ }^{20}$ M. Beruete, M. Navarro-Cía, S. A. Kuznetsov, and M. Sorolla, Appl. Phys. Lett. 98, 014106 (2011)

${ }^{21}$ M. Beruete, M. Navarro-Cía, and M. Sorolla, IEEE Trans. Microwave Theory Tech. 59, 2180 (2011).

${ }^{22}$ N. Behdad, M. Al-Joumayly, and M. Salehi, IEEE Trans. Antennas Propag. 57, 460 (2009).

${ }^{23}$ N. Behdad and M. Al-Joumayly, IEEE Trans. Antennas Propag. 58, 2460 (2010).

${ }^{24}$ C. S. R. Kaipa, A. B. Yakovlev, F. Medina, F. Mesa, C. A. M. Butler, and A. P. Hibbins, Opt. Express 18, 13309 (2010). 
${ }^{25}$ C. A. M. Butler, J. Parsons, J. R. Sambles, A. P. Hibbins, and P. A. Hobson, Appl. Phys. Lett. 95, 174101 (2009).

${ }^{26}$ M. R. Gadsdon, J. Parsons, and J. R. Sambles, J. Opt. Soc. Am. B 26, 734 (2009).

${ }^{27}$ See http://www.ansoft.com for "HFSS: High Frequency Structure Simulator Based on the Finite Element Method."
${ }^{28}$ R. B. Adler, L. J. Chu, and R. M. Fano, Electromagnetic Energy Transmission and Radiation (John Wiley \& Sons, 1960).

${ }^{29}$ O. Luukkonen, C. Simovski, G. Granet, G. Goussetis, D. Lioubtchenko, A. V. Raisanen, and S. A. Tretyakov, IEEE Trans. Antennas Propag. 56, 1624 (2008).

${ }^{30}$ D. M. Pozar, Microwave Engineering, 3rd ed. (John Wiley \& Sons, 2004). 